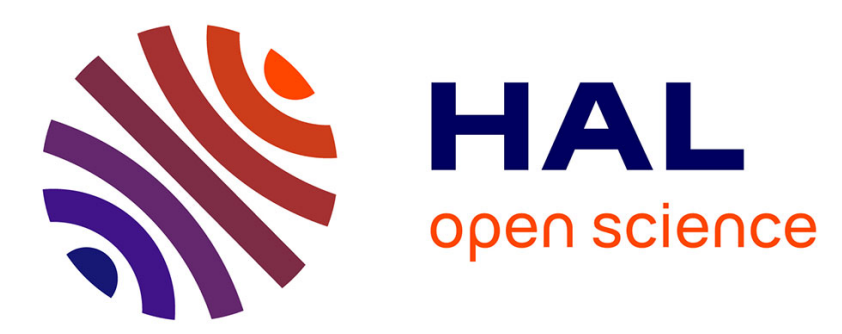

\title{
Kinked crack paths in polycarbonate samples printed by fused deposition modelling using criss-cross patterns
}

\author{
Thomas Corre, Veronique Lazarus
}

\section{To cite this version:}

Thomas Corre, Veronique Lazarus. Kinked crack paths in polycarbonate samples printed by fused deposition modelling using criss-cross patterns. International Journal of Fracture, 2021, pp.19-31. 10.1007/s10704-021-00518-x . hal-03406973

\section{HAL Id: hal-03406973 \\ https://hal.science/hal-03406973}

Submitted on 28 Oct 2021

HAL is a multi-disciplinary open access archive for the deposit and dissemination of scientific research documents, whether they are published or not. The documents may come from teaching and research institutions in France or abroad, or from public or private research centers.
L'archive ouverte pluridisciplinaire HAL, est destinée au dépôt et à la diffusion de documents scientifiques de niveau recherche, publiés ou non, émanant des établissements d'enseignement et de recherche français ou étrangers, des laboratoires publics ou privés. 


\title{
Kinked crack paths in polycarbonate samples printed by fused deposition modelling using criss-cross patterns.
}

\author{
Thomas Corre • Véronique Lazarus
}

Received: date / Accepted: date

\begin{abstract}
Additive manufacturing is unquestionably gaining importance in industry. Due to the layer by layer deposit process, it usually leads to an anisotropic material. A question of importance to assess their resistance to fracture is whether Linear Elastic Fracture Mechanics can be used. In this paper, we investigate this point on polycarbonate printed by Fused Deposition Modelling focusing on a criss-crossed deposit pattern. Thanks to tensile and fracture experiments instrumented by Digital Image Correlation, the material is evidenced to be linear elastic until fracture, nearly isotropic in the $2 \mathrm{D}$ printing plane but with a strong fracture anisotropy, leading to systematic crack kinking along the weakest plane. The Stress Intensity Factors evolution is measured across the kink and shown to be in agreement with Amestoy-Leblond's formula. The fracture toughness is observed to be larger than the bulk value, in agreement with irreversible damage and plasticity that are clearly observable at the scale of the threads.
\end{abstract}

Keywords Additive Manufacturing - Linear Elastic Fracture Mechanics - Strong fracture anisotropy . Fused Deposit Modelling (FDM)

\section{T. Corre}

IMSIA, CNRS, EDF, CEA, ENSTA Paris, Institut Polytechnique de Paris, 828 bd des Maréchaux, 91762 Palaiseau cedex, France. E-mail: thomas.corre@ensta-paris.fr

V. Lazarus

IMSIA, CNRS, EDF, CEA, ENSTA Paris, Institut Polytechnique de Paris, 828 bd des Maréchaux, 91762 Palaiseau cedex, France. E-mail: veronique.lazarus@ensta-paris.fr

\section{Introduction}

Additive manufacturing is gaining importance in industry, in particular among biomedical aerospace or building industries (Ngo et al., 2018). As layer by layer process, additive manufacturing induces specific microstructures often leading to anisotropic properties whether the bulk material is a metal, a ceramic or a polymer (Balit et al., 2020; Nguejio et al., 2019; Kok et al., 2018; Zareiyan and Khoshnevis, 2017; Ziemian et al., 2012). Such process can be used to produce composite material with tailored heterogeneous or anisotropic properties, as illustrated by advances in the manufacturing of material with bioinspired microstructure (Dimas et al., 2013; Martin et al., 2015). Recent studies took advantages of these capabilities to investigates the interactions between a crack and such precisely controlled microstructure made of different materials (Wang and Xia, 2016) or distributed inclusions (Brodnik et al., 2020). However, this anisotropy is generally not intended for conventional structural applications and understanding and controlling its appearance is a focus of additive manufacturing development (Ngo et al., 2018). In this case, it is widely analysed in terms of uniaxial properties - yield strength and strain at break (Zou et al., 2016; Song et al., 2017) - with few interest in crack propagation properties, in particular in the complex crack paths that may result from the interaction with the microstructure.

We aim here to question the propagation of a crack in those anisotropic materials answering the question whether the framework of Linear Elastic Fracture Mechanics is suitable. Here we investigate this issue on polycarbonate (PC) printed by Fused Deposition Modelling (FDM) focusing on a criss-cross deposit pattern. The paper is organized as follows. Section 2 recalls some 
basis of Linear Elastic Fracture Mechanics (LEFM) and some propagation criterion in the case of anisotropic fracture properties. Section 3 is devoted to the description of the experimental methods in term of printing, characterisation tests, fracture experiments and instrumentation by Digital Image Correlation. In section 4, the experimental results are presented and discussed. Finally, we conclude in section 5 that (i) the material has isotropic elastic properties but strong anisotropic fracture properties that systematically guide the crack along a kinked path corresponding to the nearest weakest direction; (ii) Linear Elastic Fracture Mechanics applies at the scale of the specimen while dissipation marks are clearly visible at the thread scale.

\section{Linear Elastic Fracture Mechanics}

2.1 Definition of the Stress Intensity Factors (SIF)

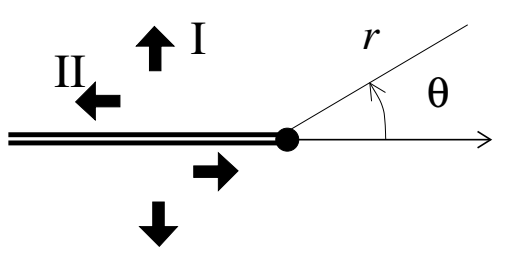

(a) Initial crack

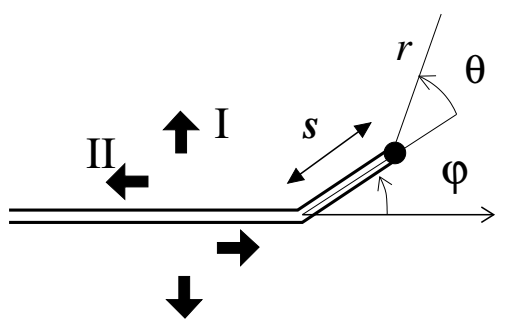

(b) With a kinked extension of length $s$ in the direction $\varphi$

Fig. 1 Zoom around the tip of a crack embedded in a linear elastic body and associated notations.

Consider a crack (fig. 1(a)) in an isotropic homogeneous elastic solid submitted to some loading (given displacements and forces). In the vicinity of its tip, the leading term of the displacement field $u$ takes the fol- lowing form in polar coordinates (Williams, 1952):

$$
\left\{\begin{aligned}
u_{r}= & \frac{K_{I}}{4 \mu} \sqrt{\frac{r}{2 \pi}}\left[(5-8 \nu) \cos \left(\frac{\theta}{2}\right)-\cos \left(\frac{3 \theta}{2}\right)\right] \\
& +\frac{K_{I I}}{4 \mu} \sqrt{\frac{r}{2 \pi}}\left[(-5+8 \nu) \sin \left(\frac{\theta}{2}\right)+3 \sin \left(\frac{3 \theta}{2}\right)\right] \\
u_{\theta}= & \frac{K_{I}}{4 \mu} \sqrt{\frac{r}{2 \pi}}\left[(-7+8 \nu) \sin \left(\frac{\theta}{2}\right)+\sin \left(\frac{3 \theta}{2}\right)\right] \\
& +\frac{K_{I I}}{4 \mu} \sqrt{\frac{r}{2 \pi}}\left[(-7+8 \nu) \cos \left(\frac{\theta}{2}\right)+3 \cos \left(\frac{3 \theta}{2}\right)\right]
\end{aligned}\right.
$$

It implies a $1 / \sqrt{r}$ singularity of the stress field when $r \rightarrow 0$, whose magnitude is given by the Stress Intensity Factors (SIF), $K_{I}$ corresponding to mode I opening loading, $K_{I I}$ to mode II plane shear loading.

The next term, proportional to $r$, involves the $T$ stress. It is linked to the dominant non-singular stress near the crack tip and can be obtained by:

$T=\lim _{r \rightarrow 0}\left[\sigma_{r r}(r, \theta=0)-\sigma_{\theta \theta}(r, \theta=0)\right]$

2.2 SIF discontinuity across a kink,

Amestoy-Leblond's formula

Assume that the loading of the body is kept constant between the two configurations of fig. 1(a) (just before kink) and fig. 1(b) (same configurations with a kinked portion of length $s$ in the direction $\varphi$ ). Denote $K_{p}$ and $T$ the SIF and the $T$-stress before the kink; $K_{p}(s, \varphi)$ the SIF defined in the same manner taking the new tip and crack direction as reference for the polar parameters (fig. 1(b)). Then in the limit $s \rightarrow 0$ :

$$
K_{p}(s, \varphi)=F_{p q}(\varphi) K_{q}+\sqrt{s} T G_{p}(\varphi)+O(s)
$$

In this equation, $F_{p q}(\varphi)$ and $G_{p}(\varphi)$ are universal functions (in the sense that they are independent of the body geometry and the applied loading), depending on the sole value of $\varphi$. This means that knowing the values of $K_{q}$ and $T$ before the kink, $K_{p}(s, \varphi)$ can in principle be obtained after the kink without the need to solve the entire elasticity problem. This remarkable result demonstrated by J.B. Leblond (1989) is valid whatever the structure considered, for isotropic and anistropic materials (Leguillon, 1993), in 2D and 3D situations (Leblond, 1999; Leblond et al., 1999; Lazarus et al., 2001). However, the value of $F_{p q}(\varphi)$ and $G_{p}(\varphi)$ differs: For an isotropic material, these functions have been determined by Amestoy and Leblond (1992); For an anisotropic material, they have not been determined yet, to the best of our knowledge.

In the case of isotropic elasticity, the values are provided by Amestoy and Leblond (1992) for any values of 
$\varphi$, either under the form of some integral equations coupled with Anderson's formula (equations $(34)_{1},(35)_{1}$, (36), (39) in there) or of a serie (equation (66) in there). In the sequel, we use the solution of the integral equations, but it is almost indistinguishable from the series (see Appendix A for datas and comparison).

Introduce the limit $K_{p}^{*}(\varphi)$ for $s \rightarrow 0$ :

$K_{p}^{*}(\varphi)=\lim _{s \rightarrow 0} K_{p}(s, \varphi)$

Using equation (3), we get

$K_{p}^{*}=F_{p q}(\varphi) K_{q}$

Note that despite the continuity of the displacement and stress fields when $s \rightarrow 0, K_{p}^{*}(\varphi)$ is discontinuous crossing the kink, that is $K_{p}^{*}(\varphi) \neq K_{p}$.

The energy release rate $G(s, \varphi)$ corresponding to a kinked propagation in the direction $\varphi$ can be obtained from the $K_{p}(s, \varphi)$ using Irwin's formula (Irwin, 1957):

$G(s, \varphi)=\frac{1-\nu^{2}}{E}\left(\left(K_{I}(s, \varphi)\right)^{2}+\left(K_{I I}(s, \varphi)\right)^{2}\right)$,

written here for $K_{I I I}=0$.

\subsection{Propagation criterion}

For anisotropic fracture properties, the criterion which emerges (Takei et al., 2013; Li and Maurini, 2019) is the Generalization of the Maximum Energy Release Rate (GMERR) (Palaniswamy and Knauss, 1975; Chambolle et al., 2009). It is a balance between the elastic energy available for propagation and the fracture costs, more precisely between the energy release rate $G^{*}(\varphi)$ (limit of $G(s, \varphi)$ for $s \rightarrow 0)$ and the surface fracture energy $G_{c}(\varphi)$. It states

1. that the propagation direction $\varphi$ is selected by the maximum of the ratio $G^{*}\left(\varphi^{\prime}\right) / G_{c}\left(\varphi^{\prime}\right)$ with respect to $\varphi^{\prime}$;

2. propagation occurs if the loading verifies $G^{*}(\varphi)=$ $G_{c}(\varphi)$, where $G^{*}(\varphi)$ is the energy released when the crack propagates in the direction $\varphi$.

Two cases are usually distinguished (Takei et al., 2013; Li et al., 2015; Li and Maurini, 2019): weak or strong anisotropy. Weak anisotropy corresponds to a convex $1 / G_{c}(\varphi)$ and strong anisotropy a non-convex one. In the weak case, the criterion reduces to a configurational torque balance (Hakim and Karma, 2009) and all material propagation directions may be observed. In the strong case, only the vicinity of the weakest planes can be reached and some forbidden directions exists.
Wulff plot inspired from cristallography can be used to conveniently describe the fundamental difference between both (Takei et al., 2013; Ibarra et al., 2016). It consists on the comparison of the polar plots of $1 / G_{c}(\varphi)$ and $1 / G^{*}(\varphi)$, propagation occurring at intersection points of both. Let us illustrate this under some assumptions that are relevant for our experiments: (i) $K_{I I}=0$ and (i) a material with 4-fold cubic symmetry, the weakest plane being oriented in the directions $\theta_{0}, \theta_{0}+90$, $\theta_{0}+180, \theta_{0}-90$ degrees, $\theta_{0}=0$ corresponding to the case where this orientation is aligned with the pure mode I direction. In this case, the energy release rate is

$G^{*}(\varphi)=\frac{E}{1-\nu^{2}} K_{I}^{2}\left(F_{I, I}^{2}(\varphi)+F_{I I, I}^{2}(\varphi)\right)$

In Figure 2, $1 / G^{*}(\varphi)$ is plotted adjusting the loading to intersect the convex $1 / G_{c}(\varphi)$ corresponding to weak anisotropy; an example of non-convex strong anisotropy is also given. In Figure 2(a), $\theta_{0}=0$ and the crack propagates in the weakest plane $\left(\varphi=\theta_{0}=0\right)$ for both weak and strong anisotropy. In Figure $2(\mathrm{~b}), \theta_{0}=35$ degrees, the crack propagates with $\varphi<\theta_{0}$ for weak anisotropy and $\varphi$ close to $\theta_{0}$ for strong anisotropy as $1 / G^{*}$ cannot reach the non-convex part of $1 / G_{c}$. In this second case, the fracture plane is always close to the weakest plane.

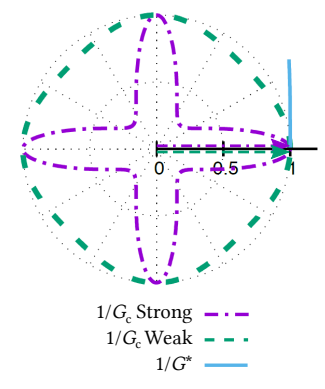

(a) $\theta_{0}=0$

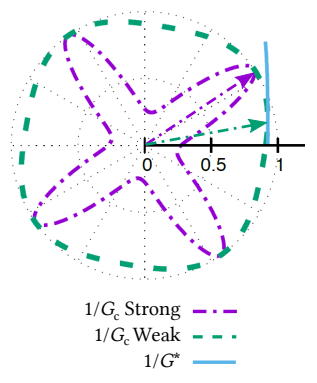

(b) $\theta_{0}=35$ degrees
Fig. 2 Wulff diagram: polar plot of $1 / G^{*}(\varphi)$ versus $1 / G_{c}(\varphi)$ for strong or weak anisotropy, given here under dimensionless form. The equilibrium point is located at the intersection $1 / G^{*}(\varphi)$ and $1 / G_{c}(\varphi)$, that gives the propagation direction corresponding to the arrows. (a) $\theta_{0}=0$, the weakest plane is aligned with the notch, the crack propagates straight in both cases. (b) $\theta_{0} \neq 0$, crack advance results from a balance between elastic and fracture energies. The green arrow is for the equilibrium in the weak case, the purple in the strong one (note that $1 / G^{*}(\varphi)$ is not plotted in this case for the sake of clarity, it would be a line parallel to the blue one passing through the tip of this arrow). When $\theta_{0}$ varies, this direction can be located anywhere for weak anisotropy, but only close to the weakest plane for strong anisotropy. 


\section{Experimental methods}

\subsection{Sample preparation}

Fused deposition modelling is a widely used additive manufacturing technique for thermoplastic polymers. In this process, the sample is built layer by layer: a feedstock filament is extruded into a heated nozzle at semiliquid state and deposited over the previously build layer (see Figure 3(a)). The extruded thread cools down,

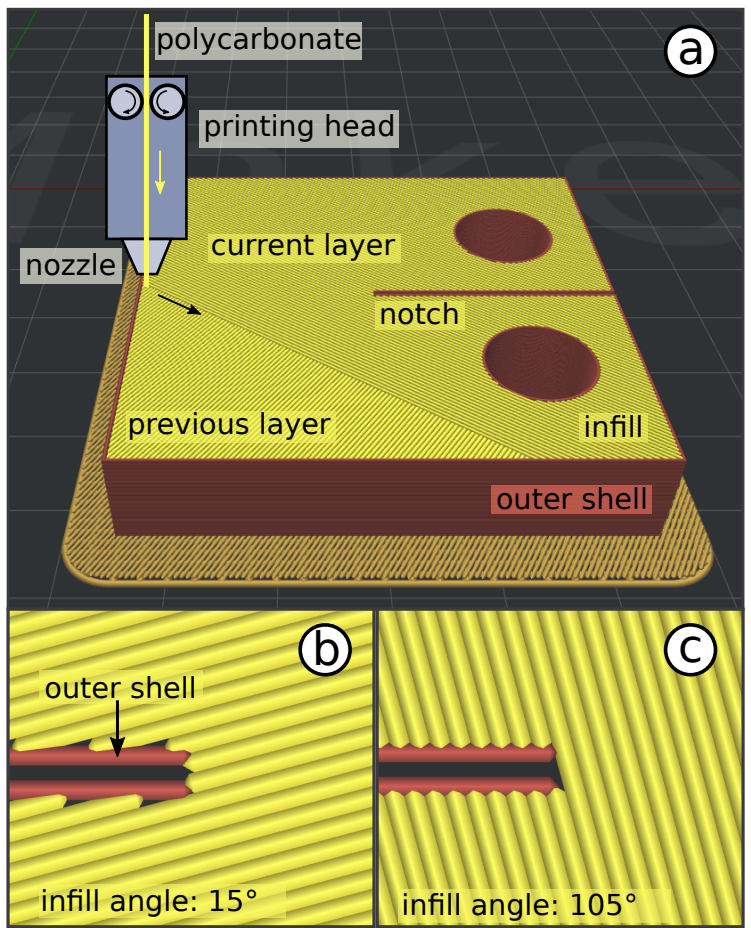

Fig. 3 (a) Representation of the trajectories of the printing head to build a CT sample (software: IdeaMaker). Details of the thread deposit around the notch: successive layers with a raster angle of (b) $15^{\circ}$ and (c) $105^{\circ}$.

solidifies and bonds to the adjacent ones. The diameter of the extrusion nozzle and the layer thickness, respectively $0.4 \mathrm{~mm}$ and $0.15 \mathrm{~mm}$ in our case, provides the approximate dimensions of the thread.

All specimens where produced in polycarbonate (PCPlus $^{\mathrm{TM}}$ from Polymaker) using a FDM 3D-printer (Raise 3D N2 plus). Each layer is filled up to $100 \%$ following a raster pattern : the threads form straight lines in the $(x, y)$ plane. Successive layers are orthogonal to each other forming a criss-cross pattern, as illustrated on Fig 3(b,c). Compact Tensile (CT) samples are used to study fracture properties and flat tensile specimens to study the material constitutive behaviour. Their geometry is detailed on Figure 4. The pattern angle is measured with respect to the notch for the Compact
Tension (CT) and with respect to the loading direction for the tensile specimens. In the following, the smallest deposit angle will be referred to as the raster angle and used to identify each specimen. Thus, a specimen with a $0^{\circ}$ raster angle is build with a $0^{\circ} / 90^{\circ}$ layup (see Fig 4(a)).

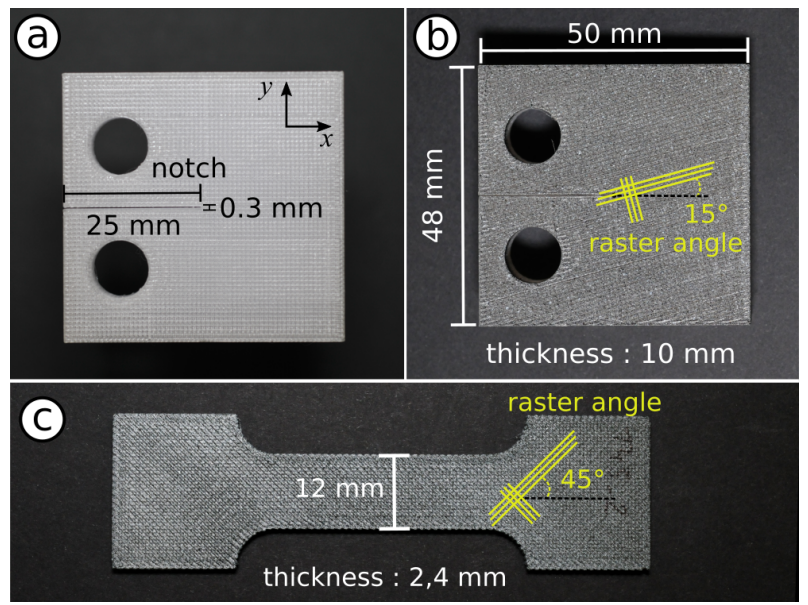

Fig. 4 (a) CT specimen with $0^{\circ}$ raster angle (without paint). Sample Geometry : (b) CT, (c) CTS and (d) tensile specimen with a speckle pattern sprayed with black and white paint.

For CT specimens, each layer is surrounded by a one-filament outer shell to ensure dimension accuracy of the $0.3 \mathrm{~mm}$ notch. However, this outer shell is removed from the notch extremity in an attempt to sharpen it. The position of the raster is adjusted so that the notch faces immediately a weld line between two threads in each layer of small raster angle, as shown on Fig 3(b). In practice, the pattern in front of the notch is disrupted by the numerous movement of the nozzle is this area. The resulting microstructure is inevitably much more complex than the theoretical one.

\subsection{Tensile tests}

The microstructure of FDM printed parts with layers made of aligned threads can induce anisotropic mechanical behaviour, as often observed (Dizon et al., 2018). The criss-cross layup exhibit 3 symmetry planes and can be described as an orthotropic material. In our case, the two deposit directions are equivalent and provide two additional symmetry planes. We consider the case of $2 \mathrm{D}$ plane stress elasticity in the $(x, y)$ plane. The compliance matrix then relies on 3 independent parameters, Young's modulus E, Poisson's ratio $\nu$ and the shear modulus $\mu$. Using Voigt notation, the generalized Hook's law with the compliance matrix $\mathbf{S}$ in the mate- 
rial basis reads:

$$
\left(\begin{array}{c}
\epsilon_{1} \\
\epsilon_{2} \\
\epsilon_{1,2}
\end{array}\right)=\left(\begin{array}{ccc}
\frac{1}{E} & \frac{-\nu}{E} & 0 \\
\frac{-\nu}{E} & \frac{1}{E} & 0 \\
0 & 0 & \frac{1}{\mu}
\end{array}\right)\left(\begin{array}{c}
\sigma_{1} \\
\sigma_{2} \\
\sigma_{1,2}
\end{array}\right)
$$

Following Ting (1996), the change of basis for the compliance matrix in Voigt notation can be expressed as:

$$
\mathbf{S}^{\prime}=\mathbf{K}^{-1} \mathbf{S K}^{-\mathbf{T}}
$$

For a rotation of the material about the third coordinate of an angle $\Theta$, the transformation matrix reduces to:

$$
\mathbf{K}^{-1}=\left(\begin{array}{cc}
\cos ^{2} \Theta & \sin ^{2} \Theta \\
\sin ^{2} \Theta & \cos ^{2} \Theta \\
\cos \Theta \sin \Theta & -\cos \Theta \sin \Theta
\end{array}\right.
$$
DIC code UFreckels (Réthoré, 2018) using a finite el$-2 \cos \Theta \sin$ fonentation of DIC with bi-linear Q4 elements

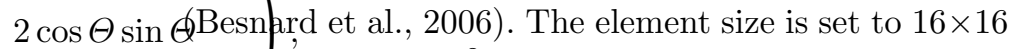
$\cos ^{2} \Theta-\sin ^{2}$ pixel $\left.320 \times 320 \mu \mathrm{m}^{2}\right)$. As shown on Figure $5(\mathrm{a})$, the dis-

The elastic modulii are then identified with tensile tests with different material orientation. Two orientations suffice in our case and experiment with $0^{\circ}$ and $45^{\circ}$ raster angle are preferred to avoid shear-coupling. Tests with $0^{\circ}$ raster angle provide $E$ and $\nu$ while tests with $45^{\circ}$ raster angle provide $\mu$.

If we further suppose that the material is isotropic in the $(x, y)$ plane (i.e. the third axis is an axis of symmetry making the material transversely isotropic), the compliance matrix keeps the form in eq. 8 in any basis and the shear modulus is related to the other parameters by the equation:

$\mu_{\text {iso }}=\frac{E_{\text {iso }}}{2\left(1+\nu_{\text {iso }}\right)}$.

In this case, tests with different material orientations are assumed to be equivalent.

Tensile tests are performed with 6 tensile specimens for each raster angle $\left(0^{\circ}\right.$ and $\left.45^{\circ}\right)$ until breakage. Plane Stress hypotheses used in formula (8) is justified by the small thickness of the specimen $(2.4 \mathrm{~mm}$ for a $48 \mathrm{~mm}$ length specimen). The test are performed with a universal tensile machine Instron $3342(30 \mathrm{kN}$ load cell) imposing a $0.5 \mathrm{~mm} \cdot \mathrm{min}^{-1}$ crosshead speed (tests up to $3 \mathrm{~mm} . \mathrm{min}^{-1}$ show no clear difference). The tests are monitored with DIC to measured the longitudinal and transverse deformation of the sample (local strains averaged over the working area of the sample). The elastic modulii are measured with a linear fit of the data up to $1 \%$ longitudinal deformation: nominal stress versus longitudinal deformation for Young's modulus and transverse strain versus longitudinal strain for Poisson's ratio. The ultimate tensile stress $\sigma_{c}$ is obtained from the nominal stress at break.

\subsection{Fracture experiments}

\subsubsection{Compact Tensile test}

Compact Tensile sample are broken on an Instron 3342 tensile machine by imposing a $0.5 \mathrm{~mm} \cdot \mathrm{min}^{-1}$ crosshead speed. A speckle pattern is sprayed over the surface of the sample using black and white paint (see Fig. 4(bd)). The test is monitored with a Nikon D300s taking greyscale images of $4288 \times 2848$ pixel every $4 \mathrm{~s}$ to $6 \mathrm{~s}$. The camera is mounted with an AF-S Micro NIKKOR $60 \mathrm{~mm}$ lens, resulting in a physical pixel size of $20 \mu \mathrm{m}$. The displacement field is measured with the open-source continuity in the displacement field highlights the crack path.

\subsubsection{Measure of $K_{p}$ using Digital Image Correlation}

Following various studies on fracture mechanics paramet al., 2009; Réthoré, 2015), the post-processing method consist in projecting the displacement field measured around the crack tip onto the Williams series. For a straight crack in a 2D elastic body, the displacement field around the crack tip takes the form of a symmetric (mode $\mathrm{I}$ ) and an anti-symmetric (mode $\mathrm{II}$ ) series (Williams, 1952):

$$
u(r, \theta)=\sum_{n=-\infty}^{\infty} a_{I}^{n} r^{\frac{1}{2}} \psi_{I}(\theta)+a_{I I}^{n} r^{\frac{1}{2}} \psi_{I I}(\theta),
$$

with $a_{I}^{n}$ and $a_{I I}^{n}$ the asymptotic coefficients (the terms for $n=1$ are detailed in eq. 1). In practice, a truncation between $n=-3$ and $n=7$ is enough to extract accurately the low order coefficients such as the stress intensity factors (Roux-Langlois et al., 2015; Réthoré, 2015). The extraction is done on a disc-like area centred around the tip of radius $r_{\text {ext }}$. A small band of width $r_{\text {int }}$ is removed from the extraction domain around the tip and along the crack lips as shown on Fig 5(b). This will reduce the influence of potential non-linear behaviour around the tip on the SIF identification. In our case, plane strain conditions are assumed since the sample width is $10 \mathrm{~mm}$. The tests are processed as systematically as possible. The extraction domain parameters are $r_{\text {ext }}=160$ pixels and $r_{\text {int }}=32$ pixels.

Following the method proposed by Hamam et al. (2007), we proceed in two steps to define the crack tip position accurately. First, using the DIC results which highlights the displacement discontinuity (Fig. 5a), we eters extraction with DIC (Besnard et al., 2006; Roux 


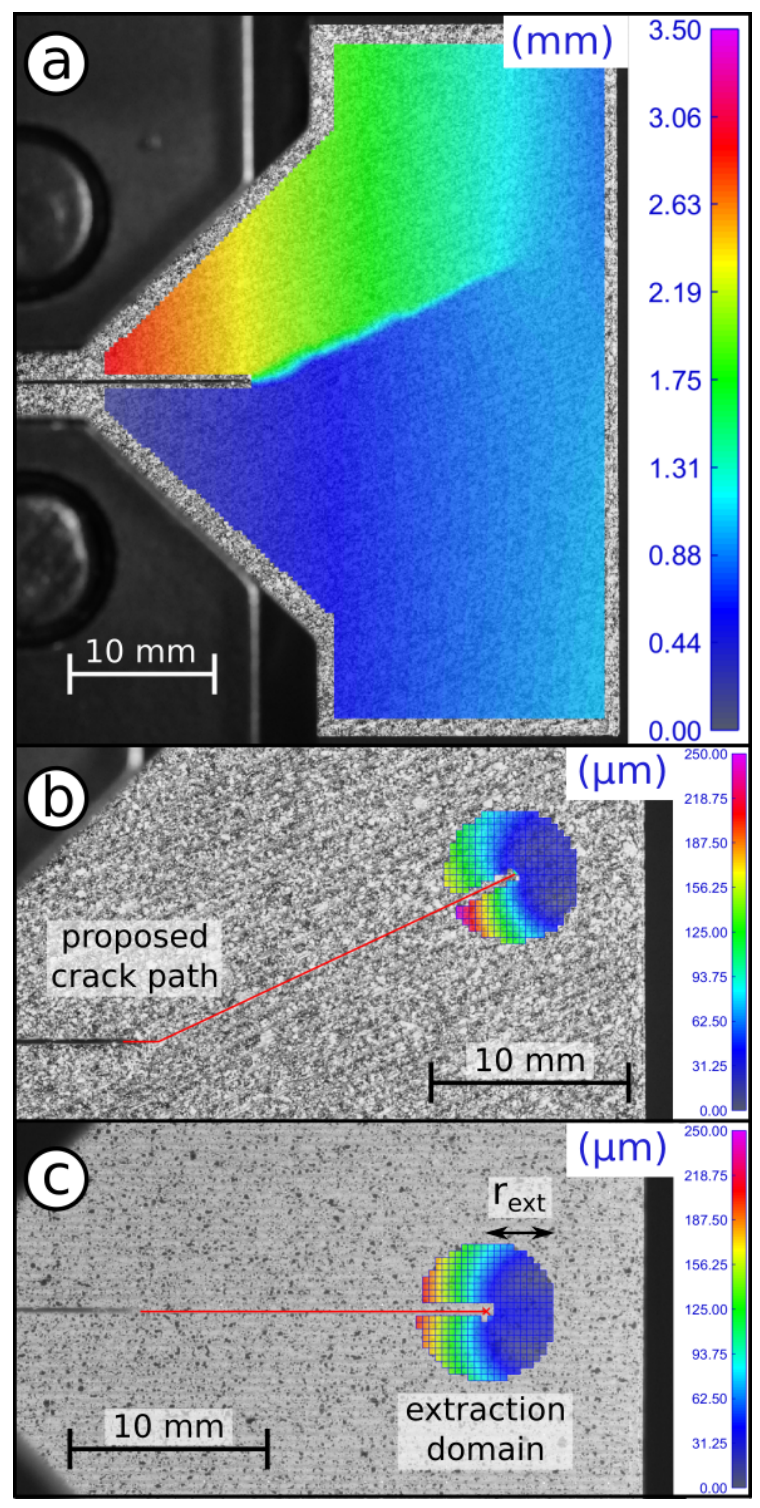

Fig. 5 (a) Vertical displacement field computed from DIC on the reference configuration of sample. The discontinuity underlines the crack path and is used to propose a crack path on which the successive positions of the tip are searched. Pixel size $20 \mu \mathrm{m}$, raster angle $25^{\circ}$. Extraction domain $\left(r_{\text {ext }}=3.2\right.$ $\mathrm{mm}$ ) and proposed crack path for raster angle of (b) $25^{\circ}$ and (c) $0^{\circ}$. The displacement field (norm) without rigid body motion is represented on the extraction domain.

propose a crack path. This proposed path is always composed of two segments: the first one is the horizontal extension of the initial notch and the second one follows the weld line between the thread which is followed by the crack as shown on Fig 6(b). As this last line is not necessarily the first one to the notch tip, the intersection of these two lines pinpoints the kink rather arbitrarily considering the complexity of the fracture process in the area ahead of the notch. However its exact location is not crucial since no SIF extraction can be done at this point : the Williams series being defined for a straight crack, the presence of a kink in the extraction domain prevent any accurate identification of the asymptotic coefficients. Second, the $n=-1$ term is used to detect more precisely the equivalent elastic crack tip position along the proposed line.

\section{Results}

\subsection{Elastic properties}

Table 4.1 presents the elastic moduli identified from the tensile tests under the assumption of orthotropic and isotropic material behaviour. The elastic parame-

\begin{tabular}{|c|c|c|c|c|c|}
\hline & \multicolumn{2}{|c|}{ orthotropic } & \multicolumn{2}{c|}{ isotropic } & \\
& mean & std & mean & std & ratio \\
\hline$E(\mathrm{MPa})$ & 1994 & 69 & 1982 & 86 & 1.01 \\
\hline$\nu$ & 0.25 & 0.03 & 0.30 & 0.06 & 0.81 \\
\hline$\mu(\mathrm{MPa})$ & 727 & 37 & 762 & 45 & 0.95 \\
\hline
\end{tabular}

Table 1 Elastic material parameters and ratio between the mean values obtained under orthotropic and isotropic assumptions. In the isotropic case, $\mu$ is computed from eq.11

ters are very close between the two models. In particular, the shear modulus in the anisotropic case is only $5 \%$ lower than the shear modulus expected from an isotropic modelling. Young modulii are equivalent and the larger discrepancy in Poisson's coefficient may come from the dispersion of the measurements. Thus, a simple isotropic model seems adequate enough to characterise the apparent 2D elastic behaviour of our polycarbonate layup. This result is consistent with previous observations on ABS (Zou et al., 2016) and polycarbonate samples printed with a criss-cross pattern (DomingoEspin et al., 2015). The isotropic material model will be used in the following. The ultimate tensile stress average is $43 \mathrm{MPa}$ with a standard deviation over every raster angle is 2.7 $\mathrm{MPa}$. We noticed a dependance on the raster orientation as commonly reported (DomingoEspin et al., 2015). However we didn't deepen this point since this mean value is sufficient for our discussion in this study.

\subsection{Fracture of the CT sample}

\subsubsection{Description}

The crack paths in two $\mathrm{CT}$ tests with different raster angles are presented on Figure 6(a-b). In every case, the 

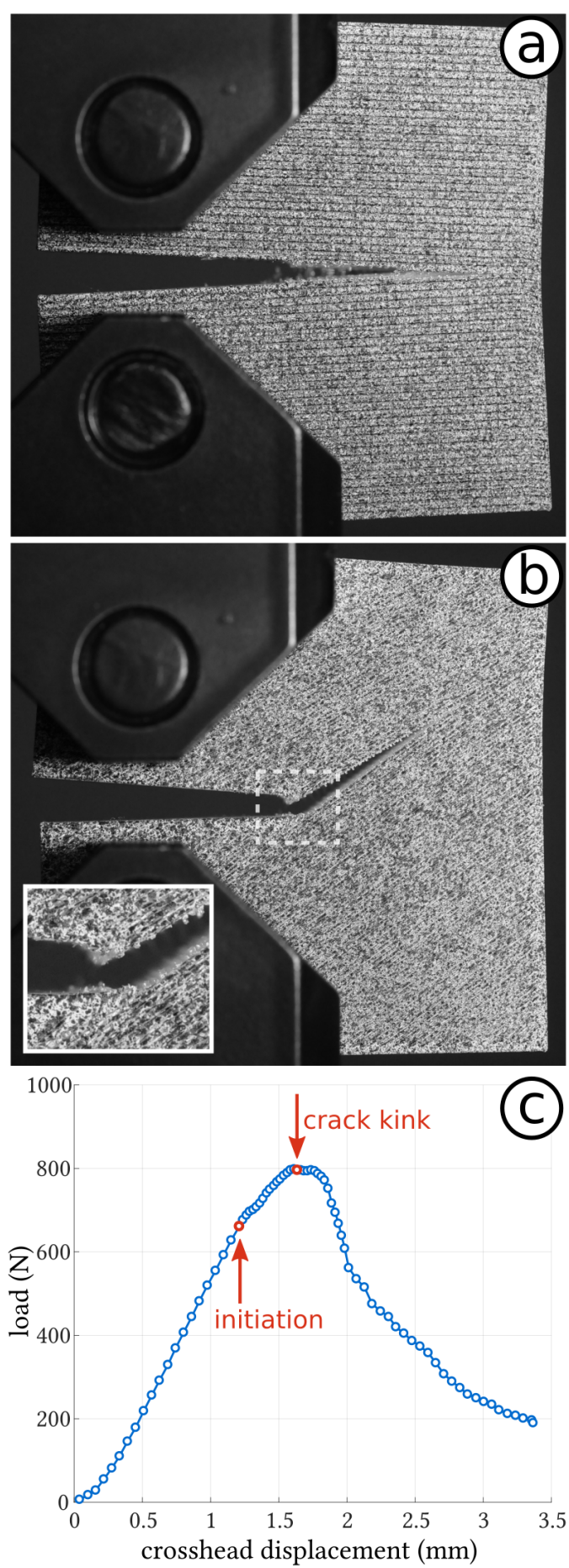

Fig. 6 Crack propagation sample with a raster angle of (a) $0^{\circ}$ and (b) $35^{\circ}$ (with zoom on the kink). (c) Typical loaddisplacement curve of the CT test, corresponding to the sample shown in (b). The approximate kinking point is highlighted in red. The initiation arrow indicates when the equivalent elastic crack tip is found about $200 \mu \mathrm{m}$ (10 pixels) away from its initial position.

same general behaviour is observed : the crack always propagates along the thread deposit direction making the smallest angle with the notch, i.e. the crack kinks with an angle equal to the raster angle. A straight crack is observed for test with a $0^{\circ}$ raster. Because of the orthogonal layup, the crack propagates along the weld line between two threads in half of the layers and perpendicularly to the threads in the other half. The fracture plan is then always the same from a microstructure point of view.

More specifically, the fracture starts with the progressive formation of a sharp crack from the printed notch. This phenomenon appears as a non-linear behaviour on the load-displacement curves, as indicated by the arrows on Fig 6(c). This initiation process is similar to a short straight propagation ahead of the notch before kinking. Accordingly, the crack plane after the kink does not correspond to the weld line that starts right ahead of the notch as noticeable on the inset on Fig 6(b)(also see Fig. 5(b)). In the following, the crack length refers to the distance traveled by the crack tip starting from the notch tip, including the short straight propagation (Fig. 1(b)).

\subsubsection{Displacement field around the tip}

The hypothesis of isotropic elasticity is further confirmed by the good agreement between the measured displacement field and the theoretical displacement provided by the Wiliams extension (eq. (1)). Indeed, the mean relative difference for each test range from $5 \%$ to $15 \%$ (displacement without rigid body motion). These global values hide to different cases: since the Williams extension (eq. (1)) is defined for a straight crack, the agreement between measured and theoretic displacement field highly depend on the presence of the kink in the extraction domain. Figure 7 shows the typical evolution of the relative difference along the crack path: the number of elements with a large relative difference decreases and remain constant once the kink left the extraction domain. Then, the difference remains low except around the lips of the crack (see inset in Fig. 7).

In this case, we plot the proportion of the extraction domain with a relative difference superior to $10 \%$ (which corresponds to an absolute difference of about $10 \mu \mathrm{m})$ to reduce the sensitivity to extremes values. This threshold is also used to evaluate the quality of extraction: the SIF measured on an image are not taken into account if more than $10 \%$ of the extraction domain exhibit a relative difference superior to $10 \%$.

\subsubsection{Evolution of $K_{p}$ during propagation for one given experiments}

The SIF for mode $I$ and mode $I I, K_{I}^{\exp }$ and $K_{I I}^{\exp }$, are represented along the crack path for a specific CT test 


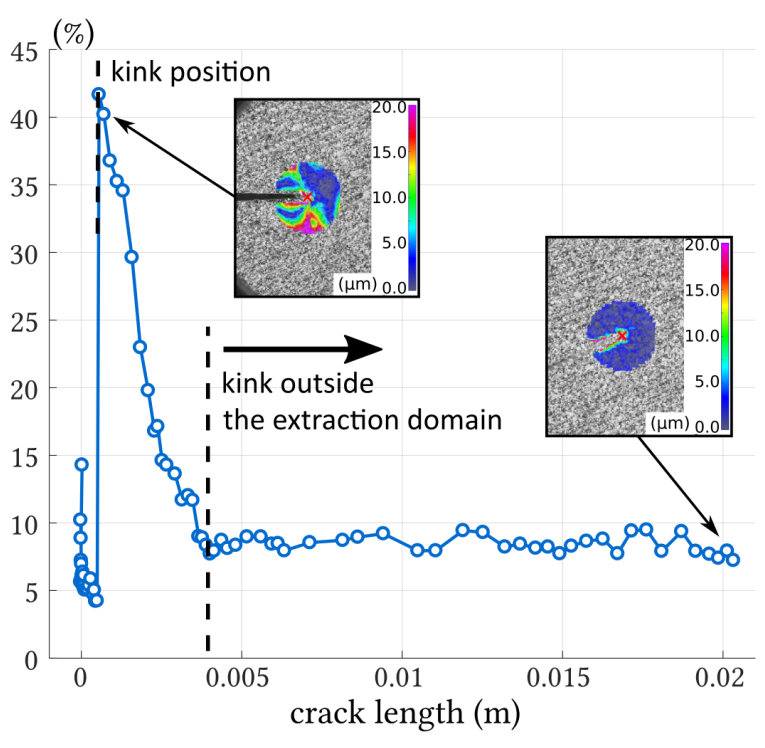

Fig. 7 Proportion of the extraction domain where the relative difference between the displacement measured and Williams extension is higher than $10 \%$. Insets show the absolute difference in two cases: just after the kink and at the end of propagation. Test with a $25^{\circ}$ kink angle (see Fig 5).

on Figure 8 for the particular cases corresponding to a raster angle of 5, 25 and 45 degrees.

The SIF measured before the kink are marked in blue: the mode $I$ loading is retrieved with $K_{I}^{\exp }$ rising and $K_{I I}^{\exp }$ approximately null. The crack length at which the crack kinks is highlighted by the jump in the value of $K_{I I}^{\exp }$ (for tests with non-zero raster angle of course). As detailed before, this kinking point is located slightly away from the notch tip (Fig. 6(b)).

Since the SIF extraction is made under the assumption of a straight crack, the kinking point has to be outside the extraction domain to obtain reliable measures. Thus, the measure of $K_{p}^{\exp }$ cannot be accurate before the crack has travelled a distance $r_{\text {ext }}=3.2 \mathrm{~mm}$ as indicated on Fig. 8.

\subsubsection{Comparison with Amestoy and Leblond Formula}

We aim here to compare the evolution of the SIF crossing the kink position, to Amestoy and Leblond formula (Eq. 5). This equation assumes that the SIF $K_{p}^{*}$ and $K_{p}$ are taken at constant loading. But the evolution of $K_{p}^{\exp }$ depicted in fig. 8 is due to changes in both the loading (fig. 6c) and the crack geometry (fig. 6b), hence can not be compared straightforwardly to Eq. 5. Moreover, Eq. 5 relies on the value of the SIF just before the kink, that are impossible to obtain with a reasonable precision, since the evolution of $K_{I}^{\exp }$ in this zone is sharp and the precise location of the kink is difficult to catch (see blue points located before the kink in fig- ure 8). We circumvent these difficulties thanks to linear elasticity, by considering the ratio $K_{I I}^{\exp } / K_{I}^{\exp }$, which is independent of (i) the SIF just before the kink and (ii) of the loading.

From Eq. 4 and Eq. 5, the limit of this ratio for $s \rightarrow 0$ is:

$\lim _{s \rightarrow 0} \frac{K_{I I}^{\exp }}{K_{I}^{\exp }}=\frac{K_{I I}^{*}}{K_{I}^{*}}=\frac{F_{I I, I}(\varphi)}{F_{I, I}(\varphi)}$.

A straightforward application of this equation is impossible experimentally since it would require measures of $K_{p}^{\exp }$ as close as possible to the kink. Instead we make the following approximation:

$\frac{K_{I I}^{*}}{K_{I}^{*}} \sim \frac{1}{s_{\text {sup }}-s_{\text {inf }}} \int_{s_{\text {inf }}}^{s_{\text {sup }}} \frac{K_{I I}^{\exp }}{K_{I}^{\exp }} \mathrm{d} s$.

We choose $s_{\text {inf }}=r_{\text {ext }}$ to eliminate the points located at a distance smaller than $r_{\text {ext }}$ of the kink position, for which the SIF can not be measured by DIC. The value of $s_{\text {sup }}$ has to be small enough so that the infinite domain assumption that is implied in the formula remains valid $^{1}$. It has also to be small enough so that the next term in the expansion $(3)$ of $K_{p}(s, \varphi)$ can be neglected. In practice, it is the case (Mesgarnejad et al., 2020) if $s \ll \mathcal{L} \equiv\left(\frac{K_{I}}{T}\right)^{2}$, where $K_{I}$ and $T$ are the values of the SIF and $T$ - stress before kink (fig. 1(a)). We choose $s_{\text {sup }}=10 \mathrm{~mm}$ as a small value in comparison to $\mathcal{L} \equiv \frac{K_{I}}{T} \sim 200 \mathrm{~mm}$ corresponding to our CT sample geometry (fig. 4$)^{2}$.

The values of $K_{I I}^{*} / K_{I I}^{*}$ obtained in this way for different kink angle are displayed on Figure 9, together with its theoretical value $\frac{F_{I I, I}(\varphi)}{F_{I, I}(\varphi)}$. We observe an overall good agreement in the whole range of kink angles studied. This further confirms the validity of the isotropic elasticity assumption and the relevance of the SIF measurements, even if several points deserves further discussion:

1. While estimation of the error of the displacement field by DIC is well known, it is not a straightforward task to report it on the values of SIF (Hamam et al., 2007). An alternative is also to take the standard deviation among the points considered in the interval, but their number is too small to make sense.

2. A natural idea to come closer to the limit $s \rightarrow 0$ is to reduce $r_{\text {ext }}$. But this decrease comes inevitably with

1 In other words, the crack tip must be far enough of the sample boundary.

2 For dimensional reasons and linearity of the problem with the loading, the value of $\mathcal{L}$ depends only on the sample geometry and can be estimated quite straightforwardly by Finite Elements. The value we obtained in this way, has been checked to be in agreement with the literature (Leevers and Radon, 1982). 

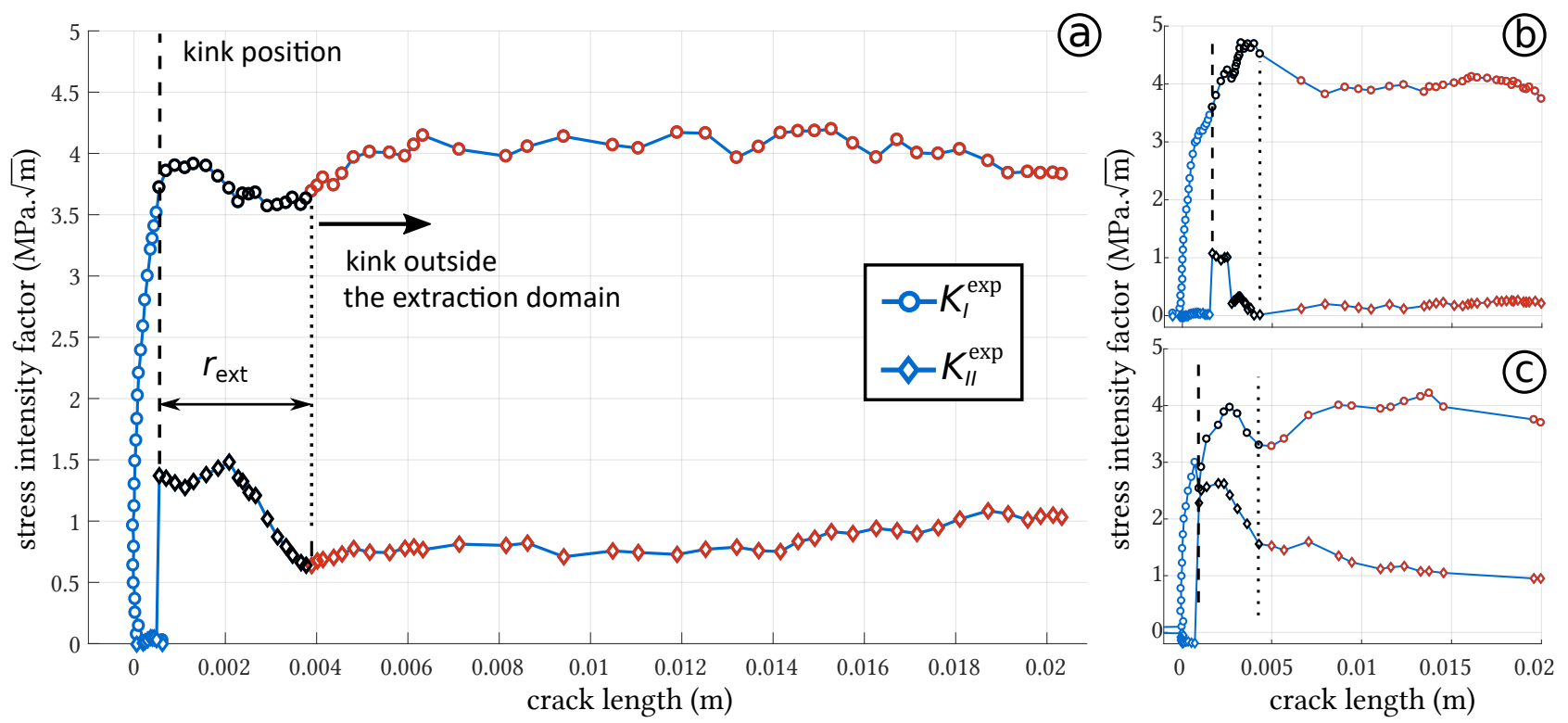

Fig. 8 Stress intensity factors obtained by DIC along crack length (measured from the notch tip). Each dot correspond to a picture where DIC is performed. Points for which $10 \%$ of the extraction domain exhibit a relative difference superior to 10 $\%$ are not represented (see section 4.2.2). Black markers indicates unreliable SIF measurements corresponding to extraction domains englobing the kink position (crack tip located at a distance smaller than $r_{\text {ext }}$ from this location). (a) $25^{\circ}$ kink angle (see Fig 5). (b) $5^{\circ}$ kink angle. (c) $45^{\circ}$ kink angle.

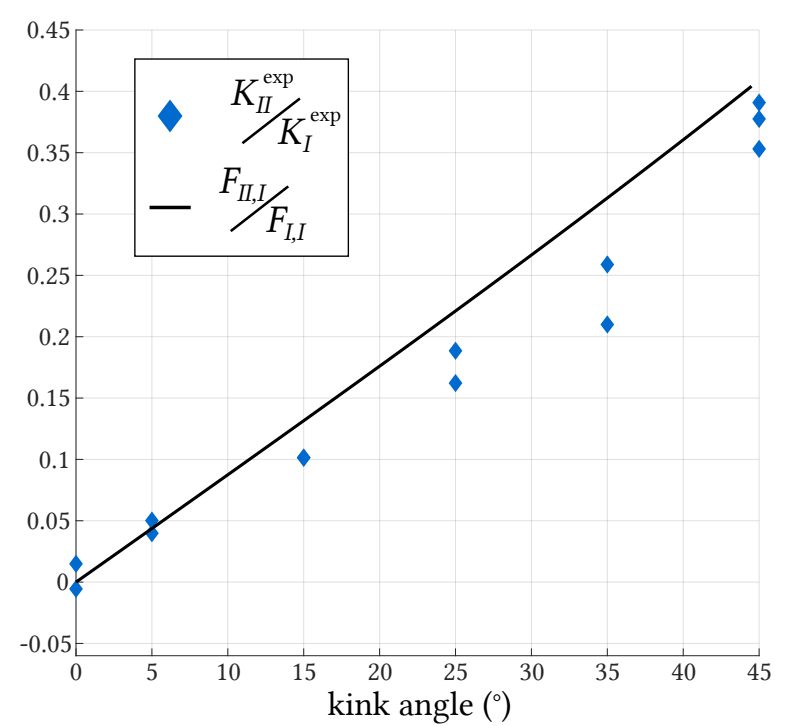

Fig. 9 Estimation of $K_{I I}^{*} / K_{I}^{*}$ for various kink angle compared to the theoretical ratio computed from Eq. 5 .

a decrease of accuracy since the extraction domain becomes smaller. It is thus not a pertinent option. We checked however that varying $r_{\text {ext }}$ between 120 and 180 pixels has a negligible effect on the values of $K_{p}^{\exp }$ and does not change the conclusion drawn from fig. 9 .

3. The tensile stress measured on tensile specimens is $43 \mathrm{MPa}$. The cohesive zone size can be estimated
(Mesgarnejad et al., 2020) to be around $0.4 G_{c} E / \sigma_{c}^{2}=$ $2.7 \mathrm{~mm}$. This is a second obstacle to use smaller values of $r_{\text {ext }}$.

4. The choice of the value of $s_{\text {sup }}$ may also be questioned. We estimated that $s_{\text {sup }}=10 \mathrm{~mm}$ is reasonable so that the crack tip does not "feel" the boundaries. We checked that moderately changing this value doesn't modify the fair agreement found in fig. 9.

5. We made a tentative to look at the second term (in $\sqrt{r}$ ) of the expansion of $K_{p}(s)$ (Eq. 3). But it appears that our setup is not suitable for this purpose. We are currently investigating this point further. It will be the purpose of another paper.

\subsubsection{Value of $G_{c}$}

The energy release rate $G^{*}$, for every crack tip position after the kink can be computed with Irwin's formula eq.(6) using previous values of $K_{p}^{*}$ and elastic constants of table. 4.1. Since crack propagation occurs Griffith threshold is reached and $G^{*}=G_{c}$. The fracture energy $G_{c}$ obtained this way shows no clear dependency to the raster angle (Figure 10(a)). This result is consistent with the crack propagating on the same fracture plan (one of the raster direction).

A critical energy release rate can be define for this plan as the mean value of $6.5 \mathrm{~kJ} \mathrm{Jm}^{-2}$ (standard deviation $600 \mathrm{~J}^{-\mathrm{m}^{-2}}$ ). This value seems superior to the 


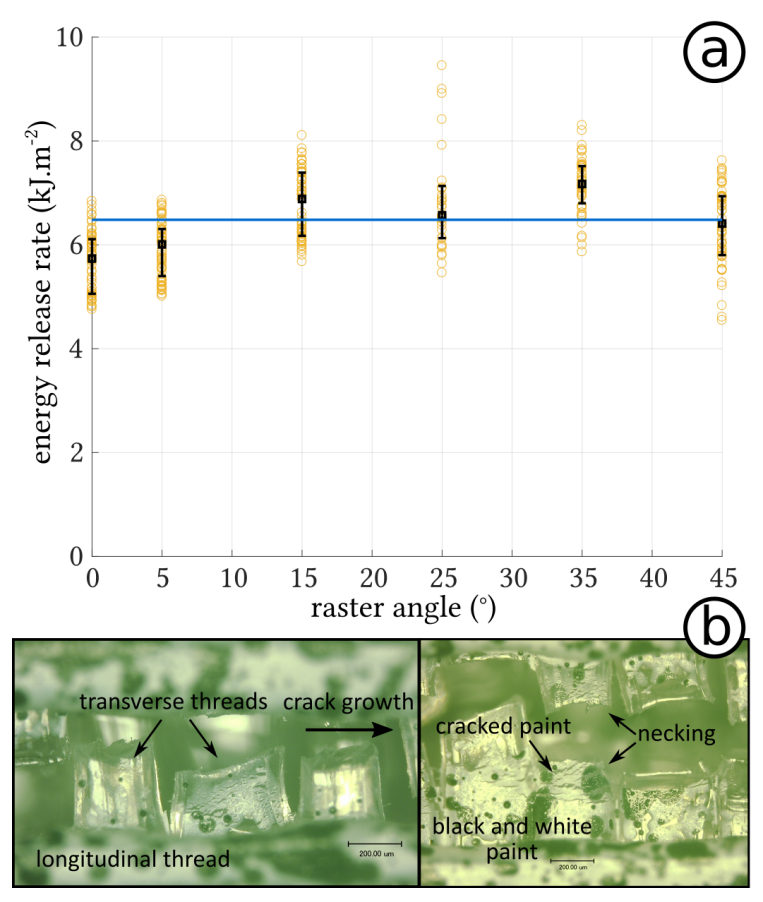

Fig. 10 (a) Energy release rate measured from every pictures used to estimate $K^{*}$. Black symbols presents first, second and third quartiles for each kink angle. Blue line is the mean over every tests. (b) Close-up on threads broken along the crack path in the first orthogonal layer. Necking of the threads and cracks in the paint reveal large local plastic deformations. (×200, opto-numerical microscope, Keyence).

energy release rate usually reported for moulded polycarbonate varying usually from $1 \mathrm{~kJ} . \mathrm{m}^{-2}$ to $5 \mathrm{~kJ} . \mathrm{m}^{-2}$ (Ashby, 1989; Kim et al., 1994). The difference might come from the large plastic deformation that polycarbonate threads undergo in layers perpendicular to the crack. A closer look at these threads on Fig 10(b) indeed reveals some necking (and some small cracks on the paint they received), which suggest plastic deformation and micro-damages. Crack trapping by voids may be another fracture toughening mechanism. Indeed, although a $100 \%$ infill is requested to the printer, optical microscopy views of the surface show incomplete adhesion between the threads. This yields some significant porosity (estimated by weighing and rough volume estimation to be around 10-15\%).

This observation recalls the inherent multi-scale fracture behaviour of these structured materials in spite of the relevant LEFM continuous description at the sample scale.

\subsubsection{Strongly anisotropic fracture toughness}

Anisotropic tensile properties of FDM printed polymers, such as the tensile strength, are well documented (Ziemian et al., 2012). However, fracture experiment often exhibit the crack path expected for isotropic material, that is for instance, straight propagation in pure mode I case. As an example, Gardan et al. (2018) observed straight crack path in CT specimen made of ABS with two different deposit layups, including a criss-cross pattern similar to ours. On the contrary, we observe a strong influence of the raster angle on the crack path for our printed polycarbonate: the crack always follow the nearest weak plane in the material corresponding to a weakly welded interface between the threads. In the framework introduced in section 2.3, it means that we have strong fracture anisotropy in our experiments. It implies that in any direction but the weakest one, the anisotropic fracture toughness is too high to be overcome by the elastic energy release rate (Li and Maurini, 2019). This hinders the possibility to propagate a crack in any other direction and implies that the critical energy release rate can only be estimated experimentally in this direction as already noticed by (Takei et al., 2013).

\section{Conclusion}

In this paper, the elastic properties and the fracture behaviour of FDM printed polycarbonate with criss-cross pattern have been studied. Compact tension tests are performed for different pattern orientation and analysed by digital image correlation. A peculiar behaviour is highlighted: the crack consistently follows a weak plane of the material corresponding to a thread deposit direction, resulting in kinked crack paths. This behaviour is the outcome of a strong anisotropic fracture toughness (Takei et al., 2013; Li and Maurini, 2019).

While strongly departing from the fracture behaviour of the isotropic bulk material, this material may be described with a careful analysis in the linear fracture mechanics framework. According to tensile tests, an isotropic elastic model seems to be accurate enough to model the behaviour of the printed material. The good agreement of the displacement field with William's expansion, used to measure the stress intensity factors, further validates this model. Leblond's formula (Leblond, 1989; Amestoy and Leblond, 1992) is retrieved experimentally in spite of the strong hypothesis necessary to estimate the close-kink limit of the stress intensity factors.

Despite the validity of LEFM observed at the continuum sample scale, polycarbonate threads orthogonal to the crack undergo large plastic deformations. This leads to surface fracture energy higher than the one usually measured for the bulk polycarbonate highlighting the inherent multi-scale fracture behaviour of these structured materials. 
Acknowledgements The authors wish to thank Julien Réthoré Hakim V, Karma A (2009) Laws of crack motion and and Raphaël Langlois for their advices on DIC. Our experimental support team, Nicolas Thurieau and Lahcène Cherfa, and the interns, Safwan Alblihed and Anis Ben Amor, are acknowledged for their help with the set up of the experiments. The support of Direction Génerale de l'Armement (DGA) and Agence de l'Innovation de la Défense (AID) is gratefully acknowledged.

\section{References}

Amestoy M, Leblond JB (1992) Crack Paths in Plane Situations - II. Detailed Form of the Expansion of the Stress Intensity Factors. International Journal of Solids and Structures 29:465-501

Ashby M (1989) Overview no. 80: On the engineering properties of materials. Acta Metallurgica 37(5):1273 $-1293$

Balit Y, Charkaluk E, and Constantinescu A (2020) Digital image correlation for microstructural analysis of deformation pattern in additively manufactured 316L thin walls. Additive Manufacturing 31:100862

Besnard G, Hild F, Roux S (2006) "finite-element" displacement fields analysis from digital images: Application to portevin-le châtelier bands. Experimental Mechanics 46(6):789-803

Brodnik N, Hsueh C-J, Faber K, Bourdin B, Ravichandran G, Bhattacharya K(2020) Guiding and trapping cracks with compliant inclusions for enhancing toughness of brittle composite materials. Journal of Applied Mechanics 87(3)

Chambolle A, Francfort G, Marigo JJ (2009) When and how do cracks propagate? Journal of the Mechanics and Physics of Solids 57(9):1614 - 1622,

Dimas L, Bratzel G, Eylon I, Buehler M (2015) Tough composites inspired by mineralized natural materials: computation, 3D printing, and testing. Advanced Functional Materials 23(36):4629-4638

Dizon JRC, Espera Jr AH, Chen Q, Advincula RC (2018) Mechanical characterization of 3D-printed polymers. Additive Manufacturing 20:44-67

Domingo-Espin M, Puigoriol-Forcada JM, GarciaGranada AA, Llumà J, Borros S, Reyes G (2015) Mechanical property characterization and simulation of fused deposition modeling polycarbonate parts. Materials \& Design 83:670-677

Gardan J, Makke A, Recho N (2018) Improving the fracture toughness of $3 \mathrm{D}$ printed thermoplastic polymers by fused deposition modeling. International Journal of Fracture 210(1-2):1-15

Gonzalez J, Lambros J (2013) Crack path selection in microstructurally tailored inhomogeneous polymers. Experimental Mechanics 53(4):619-634 phase-field models of fracture. Journal of the Mechanics and Physics of Solids 57(2):342 - 368,

Hamam R, Hild F, Roux S (2007) Stress intensity factor gauging by digital image correlation: Application in cyclic fatigue. Strain 43(3):181-192

Ibarra A, Roman B, Melo F (2016) The tearing path in a thin anisotropic sheet from two pulling points: Wulff's view. Soft Matter 12:5979-5985

Irwin GR (1957) Analysis of stresses and strains near the end of a crack traversing a plate. Journal of Applied Mechanics 24:361-364

Kim A, Bosnyak CP, Chudnovsky A (1994) Effects of weathering, scale, and rate of loading on polycarbonate fracture toughness. Journal of Applied Polymer Science 51(11):1841-1848

Kok Y, Tan XP, Wang P, Nai M, Loh NH, Liu E, Tor SB (2018) Anisotropy and heterogeneity of microstructure and mechanical properties in metal additive manufacturing: A critical review. Materials \& Design 139:565-586

Lazarus V, Leblond JB, Mouchrif SE (2001) Crack front rotation and segmentation in mixed mode I+III or I+II+III - part I: Calculation of Stress Intensity Factor. Journal of the Mechanics and Physics of Solids 49(7):1399-1420

Leblond JB (1989) Crack Paths in Plane Situations I. General Form of the Expansion of the Stress Intensity Factors. International Journal of Solids and Structures 25:1311-1325

Leblond JB (1999) Crack paths in three-dimensional elastic solids. I: Two-term expansion of the stress intensity factors-application to crack path stability in hydraulic fracturing. International Journal of Solids and Structures 36(1):79 - 103

Leblond JB, Lazarus V, Mouchrif SE (1999) Crack paths in three-dimensional elastic solids. II. Threeterm expansion of the stress intensity factors - Applications and perspectives. International Journal of Solids and Structures 36(1):105-142

Leevers P, Radon J (1982) Inherent stress biaxiality in various fracture specimen geometries. International Journal of Fracture 19:311-325

Leguillon D (1993) Asymptotic and numerical analysis of crack branching in non-isotropic materials. European Journal of Mechanics A/Solids 12:33-51

Li B, Maurini C (2019) Crack kinking in a variational phase-field model of brittle fracture with strongly anisotropic surface energy. Journal of the Mechanics and Physics of Solids 125:502-522

Li B, Peco C, Millán D, Arias I, Arroyo M (2015) Phasefield modeling and simulation of fracture in brittle materials with strongly anisotropic surface energy. 
International Journal for Numerical Methods in Engineering 102(3-4):711-727

Martin, J, Fiore B, Erb R (2015) Guiding and trapping cracks with compliant inclusions for enhancing toughness of brittle composite materials. Nature communications 6(1):1-7

Mesgarnejad A, Pan C, Erb R, Shefelbine S and Karma A (2015) Crack path selection in orientationally ordered composites. Physical Review E, 102(1),013004

Ngo TD, Kashani A, Imbalzano G, Nguyen KT, Hui D (2018) Additive manufacturing (3D printing): A review of materials, methods, applications and challenges. Composites Part B: Engineering 143:172-196

Nguejio J, Szmytka F, Hallais S, Tanguy A, Nardone S, Godino Martinez M, (2019) Comparison of microstructure features and mechanical properties for additive manufactured and wrought nickel alloys. Materials Science and Engineering: A 764:138214.

Palaniswamy K, Knauss WG (1975) Crack extension in brittle solids. Mechanics Today, Pergamon Press 4:87

Réthoré J (2015) Automatic crack tip detection and stress intensity factors estimation of curved cracks from digital images. International Journal for Numerical Methods in Engineering 103(7):516-534,

Réthoré J (2018) Ufreckles. DOI 10.5281/zenodo.1433776, URL https://doi.org/10.5281/zenodo.1433776

Roux S, Réthoré J, Hild F (2009) Digital image correlation and fracture: an advanced technique for estimating stress intensity factors of $2 \mathrm{D}$ and $3 \mathrm{D}$ cracks. Journal of Physics D: Applied Physics 42(21),

Roux-Langlois C, Gravouil A, Baietto MC, Réthoré J, Mathieu F, Hild F, Roux S (2015) DIC identification and X-FEM simulation of fatigue crack growth based on the Williams' series. International Journal of Solids and Structures 53:38-47

Song Y, Li Y, Song W, Yee K, Lee KY, Tagarielli VL (2017) Measurements of the mechanical response of unidirectional 3D-printed pla. Materials \& Design 123:154-164

Takei A, Roman B, Bico J, Hamm E, Melo F (2013) Forbidden directions for the fracture of thin anisotropic sheets: An analogy with the wulff plot. Phys Rev Lett 110:144301

Ting TCt (1996) Anisotropic elasticity: theory and applications. 45, Oxford University Press on Demand.

Williams ML (1952) Stress singularities resulting from various boundary conditions in angular corners of plates in extension. Journal of Applied Mechanics 19:526

Wang N, and Xia S (2017) Cohesive fracture of elastically heterogeneous materials: an integrative modeling and experimental study. Journal of the Mechanics and Physics of Solids 98:87-105

Zareiyan B, Khoshnevis B (2017) Interlayer adhesion and strength of structures in contour crafting-effects of aggregate size, extrusion rate, and layer thickness. Automation in Construction 81:112-121

Ziemian C, Sharma M, Ziemian S (2012) Anisotropic mechanical properties of abs parts fabricated by fused deposition modelling. Mechanical engineering 23

Zou R, Xia Y, Liu S, Hu P, Hou W, Hu Q, Shan C (2016) Isotropic and anisotropic elasticity and yielding of $3 \mathrm{D}$ printed material. Composites Part B: Engineering 99:506-513

\section{A Numerical values of the function $F_{p q}(\varphi)$ for an isotropic material}

The paper of Amestoy and Leblond (1992) provides two methods to obtain the values of $F_{p q}(\varphi)$ : the first is a semi-analytical method in which their values can be obtained by solving some integral equations coupled with Anderson's formula (equations $(34)_{1},(35)_{1},(36),(39)$ in there); the second is to use their expression under the form of a 21 terms-serie of $m=$ $\varphi / \pi$ (equation (66) in there).

The values we used here are those obtained by the first method. They can be downloaded as supplemental material. But the series provided in Amestoy and Leblond (1992) should have been used as well without any change in our final conclusions, as both methods yield (without surprise) quiet exactly the same values (see figure 11).

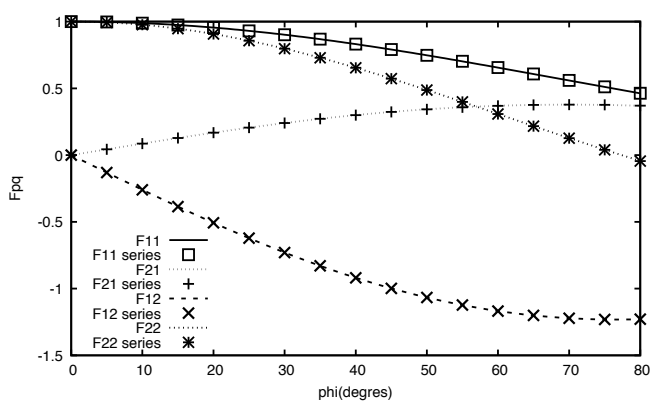

Fig. 11 Universal Functions $F_{p q}(\varphi)$ obtained by two different methods from Amestoy and Leblond (1992) 